Korean J. Math. 22 (2014), No. 1, pp. 169-180

http://dx.doi.org/10.11568/kjm.2014.22.1.169

\title{
A STUDY ON LIMITS TEACHING IN THE COLLEGE ANALYSIS MAJOR
}

\author{
Hye Young OH
}

\begin{abstract}
In this study, we consider the informal and formal definition of limit on the basis of middle and high school curriculum, and then analyze the reason of difficulties experienced when sophomores learn the formal definition $(\epsilon-\delta$ procedure) of limit. We conducted teaching of the formal definition of limit with sophomores and analyzed their errors which were appeared when they applied to limits problems. In addition, we try to improve the understanding of $\epsilon-\delta$ procedure of the limit taught in analysis.
\end{abstract}

\section{Introduction}

Analysis consists of those branches of mathematics allied to and arising from the calculus. Such studies as the calculus itself, the theory of differential equations, functional theory, the theory of infinite series, and generalized theories of integration, the calculus of variations, and so on, come under analysis[4]. Here, we will designate analysis as the subject which sophomores of the department of mathematics or mathematic eduction learn. Analysis is an essential prerequisite subject in understanding complex analysis and differential geometry as the fundamental and important subject of major mathematics. However, students

Received February 17, 2013. Revised March 20, 2014. Accepted March 20, 2014. 2010 Mathematics Subject Classification: 97D70.

Key words and phrases: analysis, errors, $\epsilon-\delta$ procedure, the informal and formal definition of limit.

This work was supported by Incheon Nathional University Research Grant in 2013.

(c) The Kangwon-Kyungki Mathematical Society, 2014.

This is an Open Access article distributed under the terms of the Creative commons Attribution Non-Commercial License (http://creativecommons.org/licenses/by -nc/3.0/) which permits unrestricted non-commercial use, distribution and reproduction in any medium, provided the original work is properly cited. 
have difficulty in understanding concepts and applying because analysis is conceptual and rigorous.

Limit concept in analysis is the concept which distinguish analysis from other branch and the basic concept in understanding infinite concept. This was the problem to cause big controversy between mathematicians in the seventeenth and eighteenth.

Students face with difficulty in procedure occurred in the definition of limit concept. $\epsilon-\delta$ procedure defines the meaning of limit rigorously and is the way to systemize limit, continuity of function, and concept of differentiability. But it is a big challenge to sophomores. It is the formal definition of limit. Difficulty or misconception which suffered in learning it causes the learning slump and becomes to abandon major mathematics. Thus, to help to understand $\epsilon-\delta$ procedure, to analyze the cause of difficulty in appearing in the procedure and continual study for finding proper teaching and learning on it are needed.

There are a lot of preceding studies on high school students' misconceptions and errors in appearing in the limit concept learning $[2,9,11,13,16]$. But the study on limit concept related to college analysis teaching is insufficient.

Thus, this study compares informal definition of limit to formal definition of it, and analyzes the cause of difficulty suffered in learning the formal definition on the basis of middle and high school curriculum. We investigate on errors appeared when sophomores apply the formal definition to problems. There are colleges which deal with $\epsilon-\delta$ procedure in calculus targeting freshmen. But we try to restrict this discussion to discussion in analysis major.

\section{Theoretical Background}

\subsection{An investigation on the informal definition of limit.}

The definition of limit of function taught in high school is given [14]:

"If we make the values of $y=f(x)$ arbitrarily close to L by taking $x$ to be sufficiently close to $a$ but not equal to $a$, we say $f(x)$ converges to $L$. We say the limit of $f(x)$, as $x$ approaches $a$, equals $L$ and

$$
L=\lim _{x \rightarrow a} f(x)
$$

or $f(x) \rightarrow L$, as $x \rightarrow a . "$ 
This is the informal definition of limit. The function of this definition is based on changing quantity as describing in middle school mathematics curriculum[10]. The expression ' $x$ is sufficiently close to $a$ ' says high school curriculum confines concept of limit to limit at cluster points. So we may say the understanding of informal definition of limit concept is based on continuous motion. To think limit as continuous motion is an intuitive and dynamic view. When solving limit of general functions which dealt with in high school curriculum, dynamic view provides an intuitive approach.

But dynamic view can hinder deeper understanding of the limit concept. Beste[1] says that students relying mainly on dynamic view may result in the following difficulties for students.

Students who rely heavily on dynamic view may think that for a function to have a limit at a point, it must be continuous at that point. They doubt on having limit at a point which is not continuous. They may also think that the limit of any function at a given point equals to the function's value.

Students relying heavily on the dynamic view tend to have a mistaken limit concept like above, but it is true that the dynamic view provides an intuitive approach.

There exist functions which can't be expressed by dynamic view alone. They are functions with isolated points or special functions such as Dirichlet function. Dynamic views such as 'the value of $x$ approaches $a$ ' are inapplicable to these functions. Because this special functions don't appear until high school, informal definition of limit has no problem. But only informal definition is insufficient to explain limit concept of special function dealt with in the department of mathematics or mathematics education. Historical investigation such as the following supported this fact.

Newton found out the primitive conception of the limit idea by attending dynamic process of successive approximation of the gradient by gradients of straight lines starting from a point[3]. However, dynamic process is not rigorous that it was considerable misunderstanding and confusion at the time Newton was doing his work and for many subsequent generations. History of mathematics says that it is not rigorous to explain limit only as dynamic process. 
Thus, to understand limit concept of special functions, conversion of thinking from function concept based on changing quantity to function concept defined by correspondence with sets is required.

\subsection{An investigation on the formal definition of limit.}

Newton referred the derivative of today as "ultimate ratios in which quantities vanish". In his book Principia, he explained it as the following, and started to use the term 'limit': "Ultimate ratios in which quantities vanish are not, strictly speaking, ratios of ultimate quantities, but limits to which the ratios of these quantities, decreasing without limit, approach." [3]

But no mathematician until Cauchy and Weierstrass was able to capture the notion of a limit in a precise mathematical fashion[3]. It was not until 1821 that the Frenchman Cauchy developed the key idea of a limit. He developed an extensive theory of limits, adequate for the calculus, he was working with the notion of a dynamic approximation process[3].

The last crucial step was achieved by German Weierstrass, who provided a formal definition of the notion of a limit. He advocated a program wherein the real number system itself should first be developed logically, and then limit concept, continuity, differentiability, convergence and divergence, and integrability defined in terms of this number system. This program became known as the "arithmetization of analysis" [5]. It was achieved by introducing meticulous $\epsilon-\delta$ procedure. For the limit concept we have the stark and incisive definition[5]:

If, given any $\epsilon>0$, there exists a $\delta>0$ such that $|f(x)-L|<\epsilon$ when $0<|x-a|<\delta$, then we say $L$ is the limit of $f(x)$ as $x \rightarrow a$.

Gone are all such vague phrases as "successive values," "ultimate ratios," "taken as small as one wishes," and "approaches indefinitely close to." All that remains in this precise and unambiguous language and symbolism are real numbers, the operation of addition(and its inverse, subtraction), and the relationship "less than" (and its inverse, "greater than") [5].

This definition says nothing about any kind of dynamic process; it simply refers to the existence of numbers $\delta$ having a certain property[3]. After it has built on the foundation of the real system, based on this, they succeeded in developing calculus strictly by excluding dynamic element 
through $\epsilon-\delta, \epsilon-N$ procedure and defining algebraically. This is the formal definition of limit concept which has been used in analysis today.

Students of the department of mathematics study $\epsilon-\delta$ procedure. Because other department except the department of mathematics use general functions just like in high school, the informal definition has no problem to students of other department. But the informal definition is inadequate for formalizing strictly compatibly with other concepts to students of the department of mathematics. The department of mathematics should use limit concept as the tool for proving continuity including special function such as Dirichlet function. For this, $\epsilon-\delta$ procedure is essential.

High school curriculum defined function as the correspondence with sets[8]. But the function was just defined statically. Calculus of high school has used the concept of function based on changing quantity just like in middle school. Limit of function using dynamic concept is all right to general function. But limit of special function such as Dirichlet function should be explained by the correspondence with sets since it was not explained by dynamic concept.

The informal definition of limit uses the expression such as "As $x$ is sufficiently close to $a, f(x)$ is arbitrarily close to $L "$. On the other hand, the formal definition of limit means that $f(x)$ can be made to lie within any preassigned distance $(\epsilon)$ from $L$ by requiring that $x$ be within $a$ specified distance $\delta$ from $a$.

Beste[1] addresses the differences between the formal and informal definition:

First, words signifying motion in the informal definition are replaced with words signifying proximity in the formal definition.

Second, dynamic procedure in the informal definition is replaced with static algebraic expression in the formal definition.

Third, metaphor of continuous motion in the informal definition is replaced with the metaphor of discreteness in the formal definition.

We can observe that dynamic view in the informal definition is changed into the static view in the formal definition, from the differences between the formal and informal definition. Thus, we can know conversion of thinking from concept of function based on changing quantity to concept of function using correspondence with sets is necessary for understanding the formal definition of limit. 


\section{The process of study}

\subsection{The method of study.}

We may know the understanding of modern changed concept of function is necessary for the understanding of the formal definition, from investigation of the formal and informal definition. So we judge that students to be familiar with dynamic concept of function until high school suffer from difficulty by the gap appeared in the process to be changed from dynamic concept of function to static concept of function.

Thus we conducted teaching of the formal definition of limit with sophomores and analyzed their errors which were appeared when they applied to the problems. The objects of this research are sophomores of $\mathrm{N}$ university, who learn the formal definition of limit ( $\epsilon-\delta$ procedure) first. The period of research was from the middle of March to April. We collected the errors of $\epsilon-\delta$ procedure by using problem-solving each chapter, explanation their own solution and then observation, analyzing of exam and interview. We could find students' errors enough through depths interview, formalize errors grouping like with like, and analyze the causes of them.

The types of errors which appeared to students are as follow. First, error on universal and existential quantifier $(\forall, \exists)$

Second, error on omitting $\epsilon$ or $\delta$

Third, error on changing $\epsilon$ and $\delta$

Fourth, error on ignoring the relation of $\epsilon$ and $\delta$

Examples of error responses each type, students' reasons for those, and researcher's interpretation on cognitive structure are as follows.

(1) Error on universal and existential quantifier $(\forall, \exists)$

Students change order of existential quantifier and universal quantifier or they confuse the meaning of them.

-Example: Explain that as $x \rightarrow a, f(x) \rightarrow L$. i.e.

$$
L=\lim _{x \rightarrow a} f(x) .
$$

-Error response: For every $\epsilon>0$, there is every $\delta>0$ such that if $0<|x-a|<\delta$, then $|f(x)-L|<\epsilon$.

-Reason for this: They think since "every $\epsilon$ " and "there is a $\delta>0$ " are not defined, the terms can be changed. 
-Interpretation: Since the understanding on existential and universal quantifiers is not clear, they confused existential quantifier with universal quantifier.

(2) Error on omitting $\epsilon$ or $\delta$

There are students omitting "for every $\epsilon>0$ " or "there is a $\delta>0$ ". In addition, they omit both "for every $\epsilon>0$ " and "there is a $\delta>0$ ", and they just write "if $0<|x-a|<\delta$, then $|f(x)-L|<\epsilon$."

-Example: Let $f(x)$ be $x^{2}+2 x-5$. Prove that as $x \rightarrow 2, f(x) \rightarrow 3$.

-Error response: If $|x-2|<\delta$, then $\left|x^{2}+2 x-5-3\right|<\epsilon$.

-Response for this: Since algebraic expression is true, even if there is no $\epsilon$ or $\delta$, the answer is correct, isn't it?

-Interpretation: It is important to realize that this procedure must work for every positive number $\epsilon$, no matter how small it is chosen. But students ignore this fact.

(3) Error on changing $\epsilon$ and $\delta$

They use existential and universal quantifier correctly but use incorrect algebraic expression like "If $0<|x-a|<\epsilon$, then $|f(x)-L|<\delta$ " or they use algebraic expression correctly but they use with changing $\epsilon$ and $\delta$. -Example: Prove that $x^{3}-4 x+7 x=4$.

-Error response:(a) For every $\epsilon>0$, there is a $\delta>0$ such that if $|x-1|<\epsilon$, then $|f(x)-4|<\delta$.

-Reason for this: They think it is okay that the order of $\epsilon$ and $\delta$ is changed.

-Interpretation: Since first it challenges with $\epsilon$ and then they must be able to produce a suitable $\delta$, they should consider what happened to dependent variable. Thus the order of independent and dependent variable should be changed. Logical reversal which $\epsilon-\delta$ procedure requires is necessary for precise formulation.

-Error response:(b) For a $\delta>0$, there is every $\epsilon>0$ such that if $|x-1|<\delta$, then $|f(x)-4|<\epsilon$.

-Reason for this: They think that it is okay there is nothing but "a $\delta<0$ " and "every $\epsilon>0$ " regardless of order.

-Interpretation: First it challenges with $\epsilon$. Then they must be able to 
produce a suitable $\delta$. But they don't know the order.

-Error response:(c) For every $\delta>0$, there is a $\epsilon>0$ such that if $|x-1|<\delta$ then $|f(x)-4|<\epsilon$.

-Reason for this: The informal definition of limit concept says "As $x$ is sufficiently close to $a, f(x)$ is arbitrarily close to 4". Thus they think that $x$ happens first and then $f(x)$ happens.

-Interpretation: The $\epsilon-\delta$ procedure of limit concept says first something is happened in $f(x)$ and then in $x$. But students don't understand this order rightly. They don't know that first $\epsilon$ is given and then $\delta$ is determined.

(4) Error on ignoring the relation of $\epsilon$ and $\delta$

This is the error appeared in applying $\epsilon-\delta$ procedure to problem. They don't know that they find mathematical expression between $\epsilon$ and $\delta$ with given conditions and the range of $x$.

-Example: Prove that $\lim _{x \rightarrow 1} x^{3}-4 x^{2}+7 x=4$.

-Error response: For every $\epsilon>0$ there is a $\delta>0$ such that if $0<$ $|x-1|<\delta$, then $|x-1|<1$. so $0<x<2$. Since

$$
\left|x^{3}-4 x^{2}+7 x-4\right| \leq|x|^{3}+4|x|^{2}+7|x|+4=42 \delta,
$$

so $\delta=\epsilon / 42$.

-Reason for this: They think that the form of $x-1$ is not necessary since the range of $x$ is solved form $|x-1|<1$.

-Interpretation: To estimate suitable $\delta$ for given $\epsilon$, there should be the relation between the proximity of limit value 4 and the proximity of $x=1$. But they don't know it.

\subsection{Analysis of Result.}

Considerable intellectual training is required to decide $\delta$ for $\epsilon$ even simple function, for students feel difficulty from the gap appeared in modern concept of function. Thus we interpreted the examples and reasons for students' errors. The causes of errors are analyzed as follows.

First, there are errors appeared because prerequisites related to logic and quantifiers were not done properly. Since students don't know quantifiers properly, they can't understand strange concept rightly. Prerequisite learning loss destroys the frame to systematize and develop special interrelation of mathematical structure logically[6]. 
Second, there are errors appeared because of misunderstanding contexts by use of letters and symbols. Using symbols makes students more mixed up and difficult, though they understand when we use words in explaining $\epsilon-\delta$ procedure. The use of quantifiers such as $\forall$ and $\exists$ made them harder.

Third, there are errors appeared in the shortage of strategy to find suitable $\delta$ depending on $\epsilon$, even though they understand basic definitions. $\delta$ depends on $\epsilon$ but there is no algorithm to decide $\delta$ for given $\epsilon$. In addition, the way for $\delta$ to depend on $\epsilon$ should be estimated each problem[15]. The relation between $\epsilon$ and $\delta$ is done precisely by just understanding the order of independent and dependent variable rightly.

Based on analyzing the cause and types of errors in the procedure of $\epsilon-\delta$, we reeducated by the following teaching method.

First, symbol is related to mathematical thinking and it helps thinking. To use letters such as $\epsilon, \delta$ and symbols such as $\forall, \exists$ are so weak in concreteness that they are proper to various context. But abstract symbolic expression is regarded as mathematical object and required to be manipulated, and so it causes learners difficulty[15]. Thus to reduce errors on using symbols and letters, we induced to use symbols after having enough period with words instead of symbols.

Second, there are a lot of errors appeared in the order of $\epsilon$ and $\delta$, though we use algebraic expressions and words. Even though algebraic expression makes rigorous mathematical thinking possible, to graph is very important mathematical thinking strategy. By using graph, the relation between $\epsilon$ and $\delta$ tried to be established.

Third, to find suitable $\delta$ depending on $\epsilon$, we made students use the method solving upside down[12]. Through solving the $\epsilon-\delta$ procedure upside down, we made most of them establish the relation of $\epsilon-\delta$ not straight but proceeding from the aim.

\subsection{Analyzing students' responses after reeducation.}

After we reeducate $\epsilon-\delta$ procedure by using teaching method of postponing use of symbols, using graph, and solving upside down, we carried out a survey through e-mail. The questions of questionnaires are as follows.

(1) How do you feel when you learn $\epsilon-\delta$ procedure at first?

(2) Is it helpful in understanding the limit concept to use words instead of symbols such as $\forall, \exists$ ? 
(3) Is it helpful in building up the relation of $\epsilon-\delta$ to use graph?

(4) Is it helpful in establishing the relation of $\epsilon-\delta$ to solve upside down?

The $\epsilon-\delta$ procedure was difficult, for it is strange and different from limit learning in high school. They doubted why $\epsilon-\delta$ procedure is used in proving the problem of limit. They say that the fact $\delta$ is decided differently each problem is not understandable at first but started to understand the meaning of $\epsilon-\delta$ procedure by solving problem. In addition, they said it took long time to understand the formal definition of limit.

Mid-lower level students say that to explain by words rather than symbols are much easier. They say that symbol is simple to write down but it is confused and induced mistakes. However, mid-upper level students say that symbol is helpful in understanding the concept. They said that they can have a glance at problem solving with symbols instead of words. They said that if they used words in $\epsilon-\delta$ procedure, it would take longer time to solve the problem. It was impressive that mid-upper and mid-lower level students' responses are different. Mid-upper students' answers remind us that symbol enables gradual development of abstract structure concept.

Students who didn't understand the order and relation of $\epsilon-\delta$ well without graph became to understand easily through graph. They say that to explain with graph is in their memory and helpful in building up concept.

They say that to use "solving upside and down" is easily understandable in proving $\epsilon-\delta$ procedure. $\delta$ is different each function but "Solving up and down" is helpful in solving $\delta$. They said they didn't seem to be able to solve the limit problem without "solving upside and down". There are students who solved problems which didn't be solved directly by solving upside and down. They could understand easily why $\delta$ was like that.

\section{Conclusion}

Because $\epsilon-\delta$ procedure appeared in the definition of limit is developed similarly in sequences, differentiation, and integration, understanding of $\epsilon-\delta$ procedure plays an important role of learning of analysis. Even though informal definition is not rigorous, the informal definition of limit 
concept is enough until high school, for high school curriculum deals with only general functions. However, it is inadequate to prove theory rigorously compatibly in the department of mathematics which has been dealt with special function including Dirichlet function. The department of mathematics uses limit as tool to prove continuity including special function. $\epsilon-\delta$ procedure is essential for this procedure. But students are confused about precise and strict development in the $\epsilon-\delta$ procedure.

Researcher compared the distinction between the informal definition and the formal one on the basis of content of the curriculum of middle and high school. And then we judged that the gap of thinking appeared in the process changed from dynamic view of function to static view of the function made students' understanding of limit concept hard.

We analyzed errors appeared when sophomores learn the formal definition of limit. Teaching method after analyzing errors does not show new method of analysis but general method. Through reeducation, we tried to improve understanding of $\epsilon-\delta$ procedure.

We may teach limit concept diversely by compensating intuitive elements of limit concept through numerical approximation approach or historical investigation of limit. Because these methods can make many students prepare on $\epsilon-\delta$ procedure intuitively and be expected to be helpful in admitting rigorous limit concept, continual research for these are necessary.

We think that repeated exercise and training of $\epsilon-\delta$ procedure is necessary for understanding the formal definition of limit because it is required to conversion of thinking which changes from dynamic view to static view. We think that training with words without symbol in $\epsilon-\delta$ procedure is definitely necessary for accurate understanding of definition and complementary study on other problem is necessary, too.

\section{References}

[1] Beste Güçler, Limitless Ways to Talk about Limits: Communicating Mathematical Ideas in the Classroom, Mathematics Teacher 105 (9) (2012), 697-701.

[2] J. S. Choi, Investigation on mis-concept about secondary school mathematics, Master's thesis, Seoul National University, 2003.

[3] K. Devlin, MATHEMATICS: The Science of Patterns, Scientific American Library, 1994.

[4] H. Eves, Foundation and Fundamental Concepts of Mathematics, PWS-KENT Publishing Co., 1990. 
[5] H. Eves, Great Moments in Mathematics, The Mathematical Association of America, 1983.

[6] G. J. Han and H. S. Woo, A Search for the meaningful method of teaching for Correct Understanding of Advanced Mathematic Concepts, J. Korea Soc. Math. Ed. Ser. A: The Mathematical Education 40 (23) (2001), 241-252.

[7] J. Han, A study on high school students' mis-concept and error about limits, Master's thesis, Korea National University of Education, 1997.

[8] M. Her, et al., High school Mathematics, Joongang Publishing Co., 2009.

[9] C. H. Lee and S. J. Lee, Semiotic Analysis of students's mis-concept and error about limits, Proceedings of the 15th International Seminar on Education of Gifted Students in Mathematics, Feb. 2010, 37-54.

[10] Ministry of Education, Science and Technology, Mathematics curriculum, 2011361(8), pp30, 2011.

[11] S. H. Park, A Study on a Model of Overcoming Cognitive Obatacles Related to the Limits of Mathematical Sequences, The Journal of Educational Research in Mathematics 9 (2) (2000), 247-262.

[12] G. Polya, How to solve it, Kyo Woo Publishing Co., 2008.

[13] K. Roh, Students' images and their understanding of definitions of the limit of a sequence, Educational studies in Mathematics 69 (2008), 217-233.

[14] S. W. Whang, et al., High school Mathematics 2, Shinsago Publishing Co., 2010.

[15] J. H. Woo, Educational Foundation of School Mathematics, Seoul National University Publishing Co., 2000.

[16] J. H. Woo and S. W. Lee, Conjecture and Metaphor of School Mathematics, The Journal of Educational Research in Mathematics 12 (4) (2002), 523-542.

\author{
Hye Young Oh \\ Department of Mathematics Education \\ Incheon National University \\ Incheon 402-808, Korea \\ E-mail: hyoh@incheon.ac.kr
}

